This item was submitted to Loughborough's Research Repository by the author.

Items in Figshare are protected by copyright, with all rights reserved, unless otherwise indicated.

\title{
Metabolism and exercise during youth
}

PLEASE CITE THE PUBLISHED VERSION

http://dx.doi.org/10.1123/pes.2016-0010

PUBLISHER

(c) Human Kinetics

VERSION

AM (Accepted Manuscript)

PUBLISHER STATEMENT

This work is made available according to the conditions of the Creative Commons Attribution-NonCommercialNoDerivatives 4.0 International (CC BY-NC-ND 4.0) licence. Full details of this licence are available at: https://creativecommons.org/licenses/by-nc-nd/4.0/

\section{LICENCE}

CC BY-NC-ND 4.0

\section{REPOSITORY RECORD}

Tolfrey, Keith. 2019. "Metabolism and Exercise During Youth". figshare. https://hdl.handle.net/2134/20593. 


\title{
Metabolism and Exercise During Youth
}

\author{
Keith Tolfrey \\ Loughborough University
}

\section{Citations}

Sarzynski MA, Davidsen PK, Sung YJ, Hesselink MKC, Schrauwen P, Rice TK, Rao DC, Falciani F, Bouchard C. Genomic and transcriptomic predictors of triglyceride response to regular exercise. Brit J Sports Med. 2015; 49:1524-1531. DOI: 10.1136/bjsports-2015-095179

\begin{abstract}
Aim We performed genome-wide and transcriptome-wide profiling to identify genes and single nucleotide polymorphisms (SNPs) associated with the response of triglycerides (TG) to exercise training. Methods Plasma TG levels were measured before and after a 20-week endurance training programme in 478 white participants from the HERITAGE Family Study. Illumina HumanCNV370-Quad v3.0 BeadChips were genotyped using the Illumina BeadStation 500GX platform. Affymetrix HG-U133+2 arrays were used to quantitate gene expression levels from baseline muscle biopsies of a subset of participants ( $\mathrm{N}=52$ ). Genome- wide association study (GWAS) analysis was performed using MERLIN, while transcriptomic predictor models were developed using the R-package GALGO. Results The GWAS results showed that eight SNPs were associated with TG training-response $(\triangle T G)$ at $p<9.9 \times 10^{-6}$, while another 31 SNPs showed $p$ values $<1 \times 10^{-4}$. In multivariate regression models, the top 10 SNPs explained $32.0 \%$ of the variance in $\Delta T G$, while conditional heritability analysis showed that four SNPs statistically accounted for all of the heritability of $\triangle T$ T. A molecular signature based on the baseline expression of 11 genes predicted $27 \%$ of $\triangle T$ T in HERITAGE, which was validated in an independent study. A composite SNP score based on the top four SNPs, each from the genomic and transcriptomic analyses, was the strongest predictor of $\Delta T G\left(R^{2}=0.14, p=3.0 \times 10^{-68}\right)$. Conclusions Our results indicate that skeletal muscle transcript abundance at 11 genes and SNPs at a number of loci contribute to TG response to exercise training. Combining data from genomics and transcriptomics analyses identified a SNP-based gene signature that should be further tested in independent samples.
\end{abstract}

Whilst this research was conducted with adults, it is relevant to those working with children and adolescents (young people) because ethical constraints often mean we must draw upon research with adults to extend our understanding of potential mechanisms. Although the sample size for this study was relatively small, the data are based on biopsies of skeletal muscle, which would not be available from young people completing an exercise intervention to control potentially deleterious triacylglycerol concentrations ([TAG]). An evolving understanding of environmental influences on gene expression, allied to the development of non-invasive methods to examine genomics and transcriptomics, will soon probably lead to an increase in similar studies in young people. Elevated postprandial triacylglycerol concentrations ([TAG]) are predisposed to the development and progression of atherosclerosis (11), and independently predict future cardiovascular disease risk in adults (7). Although the clinical manifestations of atherosclerotic disease emerge in adulthood typically, the paediatric origins of atherosclerosis are well established (5). Furthermore, childhood fasting [TAG] predicts young adult cardiovascular disease risk (6). 
Most people spend the majority of waking hours in a postprandial state, resulting in extended periods of elevated postprandial [TAG]. Considering cardiovascular disease remains the leading cause of mortality worldwide (4), prevention by targeting modifiable risk factors is a high priority on the public health agenda. Therefore, lifestyle modifications that reduce postprandial [TAG] from a young age may delay precursors of atherosclerotic disease leading to important long-term metabolic health benefits (5). There is a relatively long history of research examining the effects of manipulations in exercise and diet on postprandial triacylglycerol (TAG) metabolism dating back to 1994, when Hardman's group published their first paper with healthy adults (1). Since then, Thomas, Gill, Stensel, Barrett and Tolfrey, amongst others, have each extended this work by examining some of the underlying mechanisms and exploring acute metabolic responses in children and adolescents. Gill and Tolfrey have, independently, identified considerable interindividual variability in the postprandial TAG response to exercise in adults and adolescents, respectively; Gill et al. (3) suggested that the wide ranging TAG response to exercise (-32\% to $+28 \%)$ might be underpinned by variation in hepatic fatty acid oxidation following exercise in middleaged men with type 2 diabetes. Tolfrey and colleagues found that individual variability in high intensity, short-duration exercise intensity explained $42 \%$ of the variance in exercise-induced changes in postprandial TAG in adolescent boys, which ranged from $-34 \%$ to $+16 \%$ (9); however, it has not been possible to identify other determinants of this variability in several other studies where exercise intensity has been more tightly controlled (10).

Sarzynski et al. (8) used a combination of genomic and transcriptomic profiling techniques to identify genes and single nucleotide polymorphisms (SNPs), also known as sequence variants or allelic variants, associated with exercise training-induced changes in fasting plasma TAG concentration in a sub-sample of 49 white adults from the HERITAGE Family Study. A primary driver behind this research was concern that exercise, normally considered to be "safe" for most healthy individuals, led to an adverse metabolic outcome; in this case, an increase in TAG of at least two technical errors greater than no change $(\geq 0.42 \mathrm{mmol} / \mathrm{L})$ in a mixed race/sex sample of 1,687 exercisers (2). Without prior experience of experimental genetic research, the descriptions of genome-wide association study (GWAS) SNP genotyping, affymetrix microarray analysis, baseline ribonucleic acid (RNA) gene signature and SNP summary score were challenging. Nevertheless, the possibility that this research might transfer to acute exercise postprandial lipaemia measures in young people was an exciting prospect and the main reason why this publication is featured here. Sarzynski et al. (8) found that numerous genes and SNPs contributed to the genetic and transcriptomic variation in the chronic exercise training response of TAG (i.e., 
20 weeks of training, 3.week ${ }^{-1}$ ). Specifically, four SNPs explained all of the genetic variance of the white participants' TAG response to exercise training in the HERITAGE Family Study. Using an independent study for validation, $27 \%$ of the TAG exercise response was predicted by a molecular signature based on the baseline expression levels of 11 genes; this procedure was accomplished using a multivariate variable selection based on a genetic algorithm method that seeks to identify the best subset of genes for maximising the fitness of the regression model. The authors explained that their SNP summary score, derived by combining genomics and transcriptomics, could potentially be used as an a priori predictor of a trait response to regular exercise; from this, more effective preventive or treatment pathways might be identified. However, it is recognised that these initial findings need to be validated in further independent studies before the possibility of individualised exercise training programmes could be considered realistically to manage TAG concentrations. In their discussion, the authors expand on how the genetic effect of regular exercise may be exerted through three different pathways (heparan sulfate glycosaminoglycan and glycosphingolipid biosynthesis and cell-adhesion molecules), but they note that the effect of exercise requires further clarification. Similarly, the pathway analysis showed that oxidative phosphorylation and mitochondrial dysfunction pathways were prominent transcriptomic predictor models for the TAG response to exercise training. Importantly, they concluded that the genes most important in modifying changes in fasting TAG in response to regular exercise are not the same as the loci controlling variation in population TAG concentrations. Sarzynski et al. (8) explain that, regardless of the "biological plausibility of the identified variants, identifying predictors of favourable and unfavourable TAG response to regular exercise could help to optimise therapeutic strategies" (p.1530) - this is certainly a long-term objective of ours when considering the effect of exercise (and dietary) manipulations on postprandial TAG in young people. Mindful of the various study limitations provided by the authors at the tail end of their manuscript, I am keen to see whether their findings can transfer to acute exercise models of postprandial lipaemia in young people.

\section{References}

1. Aldred HE, Perry IC, Hardman AE. The effect of a single bout of brisk walking on postprandial lipemia in normolipidemic young adults. Metabolism. 1994;43(7):836-41.

2. Bouchard C, Blair SN, Church TS, Earnest CP, Hagberg JM, Häkkinen K, Jenkins NT, Karavirta L, Kraus WE, Leon AS, Rao DC, Sarzynski MA, Skinner JS, Slentz CA, Rankinen T. Adverse metabolic response to regular exercise: is it a rare or common occurrence? PLoS One. 2012;7(5):e37887. 
3. Gill JMR, Al-Mamari A, Ferrell WR, Cleland SJ, Perry CG, Sattar N, Packard CJ, Caslake MJ, Petrie JR. Effect of prior moderate exercise on postprandial metabolism in men with type 2 diabetes: heterogeneity of responses. Atherosclerosis. 2007;194(1):134-43.

4. GBD 2013. Mortality and causes of death collaborators global, regional, and national age-sex specific all-cause and cause-specific mortality for 240 causes of death, 1990-2013: a systematic analysis for the Global Burden of Disease Study 2013. Lancet. 2015;385:117-71.

5. McGill HC, McMahan CA, Herderick EE, Malcom GT, Tracy RE, Strong JP. Origin of atherosclerosis in childhood and adolescence. Am J Clin Nutr. 2000;72(5):1307S-15S.

6. Morrison JA, Glueck CJ, Wang P. Childhood risk factors predict cardiovascular disease, impaired fasting glucose plus type 2 diabetes mellitus, and high blood pressure 26 years later at a mean age of 38 years: the Princeton-lipid research clinics follow-up study. Metabolism. 2012;61(4):531-41.

7. Nordestgaard BG, Benn M, Schnohr P, Tybjærg-Hansen A. Nonfasting triglycerides and risk of myocardial infarction, ischemic heart disease, and death in men and women. JAMA 2007;298(3):299-308.

8. Sarzynski MA, Davidsen PK, Sung YJ, Hesselink MKC, Schrauwen P, Rice TK, Rao DC, Falciani F, Bouchard C. Genomic and transcriptomic predictors of triglyceride response to regular exercise. Brit J Sports Med. 2015; 49:1524-1531.

9. Thackray AE, Barrett LA, Tolfrey K. Acute high-intensity interval running reduces postprandial lipemia in boys. Med Sci Sports Exerc. 2013;45(7):1277-84.

10. Tolfrey K, Thackray AE, Barrett LA. Acute exercise and postprandial lipemia in young people. Pediatr Exerc Sci. 2014;26(2):127-37.

11. Zilversmit DB. Atherogenesis: a postprandial phenomenon. Circulation. 1979;60(3):473-85. 


\title{
Citation
}

Bond B, Cockcroft EJ, Williams CA, Harris S, Gates PE, Jackman SR, Armstrong N, Barker AR. Two weeks of high-intensity interval training improves novel but not traditional cardiovascular disease risk factors in adolescents. Am J Physiol - Heart Circ Physiol. 309(9):H1039-H1047. DOI: 10.1152/ajpheart.00360.2015

\begin{abstract}
High-intensity interval training (HIIT) improves traditional cardiovascular disease (CVD) risk factors in adolescents, but no study has identified the influence of HIIT on endothelial and autonomic function in this group. Thirteen 13- to 14-yr-old adolescents (6 girls) completed six HIIT sessions over 2 wk. Each training session consisted of eight to ten 1-min repetitions of cycling at $90 \%$ peak power interspersed with $75 \mathrm{~s}$ of unloaded cycling. Traditional (triglycerides, cholesterol, glucose, insulin, and blood pressure) and novel [flow-mediated dilation (FMD), heart rate variability (HRV)] CVD risk factors were assessed in a fasted and postprandial state before (PRE), 1 day after (POST1D), and 3 days after (POST-3D) training. Aerobic fitness was determined PRE and POST-3D. Two weeks of HIIT had no effect on aerobic fitness or traditional CVD risk factors determined in the fasted or postprandial state $(P>0.15)$. Compared with PRE, fasted FMD was improved POST-1D $[P=0.003$, effect size $(E S)=0.70]$ but not POST-3D $(P=0.32$, ES $=0.22)$. Fasted FMD was greater POST-1D compared with POST-3D $(P=0.04$, ES $=0.48)$. Compared with PRE, postprandial FMD was greater POST-1D $(P<0.001, \mathrm{ES}=1.01)$ and POST-3D $(P=0.01, \mathrm{ES}=0.60)$. Fasted HRV was greater POST-1D $(P=0.001, \mathrm{ES}=0.71)$ and POST-3D $(P=0.02, \mathrm{ES}=0.44)$. The test meal lowered HRV in all laboratory visits $(P<0.001, \mathrm{ES}=0.59)$, but there were no differences in postprandial HRV between visits $(P>0.32$ for all). Two weeks of HIIT enhanced endothelial function and HRV without improvements in traditional CVD risk factors. However, most of this favorable adaptation was lost POST-3D, suggesting that regularly performing high-intensity exercise is needed to maintain these benefits.
\end{abstract}

This experimental study captures many features that are important when considering cardiometabolic health in adolescents and has numerous novel features worthy of inclusion in this special feature. The main premise for this research is that so-called traditional risk factors appear to account for only $\sim 60 \%$ of exercise-induced cardiovascular disease (CVD) risk reduction. Therefore, "novel" risk factors are being targeted increasingly to explain the remaining $40 \%$ proportion of variance or "risk factor gap". Bond and colleagues (1) focused on endothelial function and heart rate variability (HRV; autonomic function) as novel markers to compare and contrast with the traditional measures that included systolic blood pressure (SBP), plasma [glucose], [insulin], [triacylglycerol] ([TAG]), [total cholesterol] ([TC]), [high-density lipoproteincholesterol] ([HDL-C]) and [glutathione peroxidase] ([GTP]), which is an enzyme involved in cellular antioxidation. Whether GTP is a traditional or novel risk factor is probably open to debate. Low volume, high intensity interval training (HIIT) provided the exercise stimulus in the six training sessions spread over two weeks, which capitalises on the current vogue for this type of exercise and is similar to previously published acute studies with adolescents $(3,6)$ spawned from studies with sedentary (4) and type-2 diabetic adults (5). Another novel feature of this research was the 
inclusion of postprandial measures to overcome the limitation of previous studies that have relied on the less dynamic fasting plasma concentrations of the traditional risk factors listed above. The study was designed so that it was possible to separate the acute response, from the last exercise session, and the chronic two week training adaptation by including pre-exercise, 1-day postexercise and 3-day post-exercise measurements; however, a non-exercise matched control group was not included, but the authors suggested this is consistent with the HIIT literature. Major strengths of this study were the tight control, standardisation or quantification of objectively measured free-living physical activity and dietary intake/composition $48 \mathrm{~h}$ before each laboratory visit and the carefully supervised progressive HIIT programme.

All, but one, of the measures described under the traditional CVD risk factor sub-heading (including peak mechanical power (PP) and cardiorespiratory fitness) did not change significantly $(P \geq 0.18)$ over the two-week intervention period and the two effect sizes provided were trivial ( $d \leq 0.12)$. These results stood regardless of whether the measures were fasted or postprandial. If you are wondering what changed significantly, it was PP $(P=0.002)$, but the effect was still small $(d=0.32)$. It was not possible to discern whether the study was powered adequately to detect meaningful changes in these variables in asymptomatic, moderately fit, healthy teenage girls and boys; this is particularly pertinent when considering the three drop-outs, but most likely the participant baseline characteristics are the greatest barrier to change (a common limitation in my own research over the years). A summary of the effect sizes, to accompany the traditional probability values, would have been welcome for the metabolic measures. In contrast, statistically significant changes $(P \leq 0.04)$ in the novel risk factors (flow-mediated dilation (FMD), baseline arterial diameter and HRV) over the six training sessions, were found; effect sizes ranged from small to large ( $d=0.39$ to 0.97 ). Closer examination of the results revealed that the 1 -day postexercise effects were larger than those found 3-days after the last exercise training session (compared with the pre-exercise baseline). There were also some subtle, but noteworthy differences between fasting and postprandial measures that support the notion that the body's response to digesting a meal provides a more insightful window to metabolism than overnight fasting conditions - it was clear that test meal consumption promoted a reduction in FMD and HRV, whilst increasing plasma [TAG] across all visits compared with fasting. This is perhaps unsurprising given the test breakfast meal had a very high energy content of $7134 \mathrm{~kJ}$ ( 1704 kcal) this equates to approximately $96 \%, 91 \%$ and $82 \%$ of the samples' measured mean daily energy intakes in the $48 \mathrm{~h}$ preceding the pre, 1-day post and 3-day post laboratory visits respectively. The 
authors highlighted the primary study outcomes as: (i) a HIIT-induced improvement [sic] in endothelial function and HRV in boys and girls; (ii) changes in novel and traditional CVD risk factors may occur independently; and (iii) the changes in endothelial function and HRV were transient (\% $\Delta 1$-day $>\% \Delta 3$-day), which suggest their findings may reflect an acute response from the last exercise training bout rather than a chronic physiological or metabolic adaptation. Given the "training" was only six sessions over two weeks, this might be expected in a population where training effects have often been reported to be blunted compared with adults; however, I recognise the research design matches some features of the study that led to the recent resurgence in HIIT from Canada (2). From a public health perspective, if these results can be replicated with a larger sample of representative young people, future exercise or physical activity policies should continue to recommend that this is experienced regularly. Bond et al. (1) do indicate this may need to be repeated every day to realise the health benefits in adolescents, but I suspect the risk of repetitive strain or overuse injuries will need to be considered very carefully for young people who wish to partake only in high intensity exercise. My interpretation of the authors' perspective on HIIT for adolescents is that it is another form of exercise to choose from and may work best if blended with other intensities and durations. This may be important from a motivational standpoint and could influence long-term adherence to habitual exercise during childhood and adolescence. Finally, the authors provided some reflections on possible underlying mechanisms drawn from a variety of literature sources - this speculation can be interesting when ethical barriers to invasive procedures are precluded in young people, though it is possible some future studies may address these gaps in our knowledge.

\section{References}

1. Bond B, Cockcroft EJ, Williams CA, Harris S, Gates PE, Jackman SR, Armstrong N, Barker AR. Two weeks of high-intensity interval training improves novel but not traditional cardiovascular disease risk factors in adolescents. Am J Physiol - Heart Circ Physiol. 309(9):H1039-47.

2. Burgomaster KA, Hughes SC, Heigenhauser GJF, Bradwell SN, Gibala MJ. Six sessions of sprint interval training increases muscle oxidative potential and cycle endurance capacity in humans. J Appl Physiol. 2005;98(6):1985-90.

3. Cockcroft EJ, Williams CA, Tomlinson OW, Vlachopoulos D, Jackman SR, Armstrong N, Barker AR. High intensity interval exercise is an effective alternative to moderate intensity exercise for improving glucose tolerance and insulin sensitivity in adolescent boys. J Sci Med Sport. 2015;18:720-24. 
4. Hood MS, Little JP, Tarnopolsky MA, Myslik F, Gibala MJ. Low-volume interval training improves muscle oxidative capacity in sedentary adults. Med Sci Sports Exerc. 2011;43: 184956.

5. Little JP, Gillen JB, Percival ME, Safdar A, Tarnopolsky MA, Punthakee Z, Jung ME, Gibala MJ. Low-volume high-intensity interval training reduces hyperglycemia and increases muscle mitochondrial capacity in patients with type 2 diabetes. J Appl Physiol. 2011;111(6):1554-60.

6. Thackray $A E$, Barrett LA, Tolfrey $\mathrm{K}$. Acute high-intensity interval running reduces postprandial lipemia in boys. Med Sci Sports Exerc. 2013;45(7):1277-84.

Dr Keith Tolfrey thanks Dr Alice Thackray and James Smallcombe MSc for reviewing a draft version of this paper. 